\title{
La dialectique province-Paris dans la presse des départements : entre vie politique locale et réseaux nationaux d'opinion
}

\section{Éric Wauters}

\author{
(2) OpenEdition \\ Journals \\ Édition électronique \\ URL : https://journals.openedition.org/ahrf/758 \\ DOI : 10.4000/ahrf.758 \\ ISSN : 1952-403X \\ Éditeur : \\ Armand Colin, Société des études robespierristes \\ Édition imprimée \\ Date de publication : 1 décembre 2002 \\ Pagination : 71-85 \\ ISSN : 0003-4436 \\ Référence électronique \\ Éric Wauters, «La dialectique province-Paris dans la presse des départements : entre vie politique \\ locale et réseaux nationaux d'opinion », Annales historiques de la Révolution française [En ligne], 330 | \\ octobre-décembre 2002, mis en ligne le 17 avril 2008, consulté le 23 avril 2022. URL : http:// \\ journals.openedition.org/ahrf/758; DOI : https://doi.org/10.4000/ahrf.758
}




\title{
LA DIALECTIQUE PROVINCE-PARIS DANS LA PRESSE DES DÉPARTEMENTS : ENTRE VIE POLITIQUE LOCALE ET RÉSEAUX NATIONAUX D'OPINION
}

\author{
ÉRIC WAUTERS
}

\begin{abstract}
La circulation de l'information pendant la Révolution n'obéit pas à un simple schéma pyramidal, de la capitale vers la province. La distribution de la presse locale traduit d'abord l'influence culturelle ou économique de quelques villes. Diffusant une information essentiellement nationale, la presse tend, ensuite, à créer de nouveaux groupes qui se constituent autour des mêmes sensibilités idéologiques ou des mêmes intérêts de classes.

Comment ces groupes s'articulent-ils entre eux ou avec la capitale, pour constituer un courant d'opinion nationale? Une vaste enquête nationale sur la distribution des nouvelles, entre la capitale et la province d'une part, entre les départements d'autre part, permettrait sans doute de mieux connaître les mécanismes de formation de l'opinion publique.
\end{abstract}

Mots clés : presse ; circulation de l'information ; pratiques politiques ; opinion publique.

«Dans un moment où chaque département et chaque ville acquièrent la faculté de s'administrer publiquement, il est nécessaire que toutes les provinces entretiennent entre elles des relations fréquentes, et elles ne le peuvent faire que par la voie des journaux " (prospectus du Journal patriotique de Bourgogne, 1790).

Que l'on aborde la presse locale sous l'angle des conditions matérielles de production ou dans le champ de la circulation des idées politiques, le constat est le même : l'importance de la capitale productrice, d'une part, de journaux distribués sur tout le territoire, concurrençant, parfois jusqu'à l'éliminer, la presse locale, et, d'autre part, d'informations politiques qui, du récit événementiel aux comptes rendus des séances des assemblées, constituent l'essentiel de la matière première de tous les périodiques où qu'ils 
soient produits. Mais cette communication ne porte pas sur l'image de Paris dans la presse de province, question dont Hugh Gough avait, au Congrès mondial de 1989, esquissé les contours (1) : il n'est d'ailleurs pas certain qu'un corpus plus large que le sien, voire un travail exhaustif, apporteraient davantage à ses conclusions, si ce n'est de produire, comme toujours dans le champ de l'histoire révolutionnaire, un énorme nuancier qui se prêtera à toutes les interprétations.

On doit tout de même signaler quelques convergences, et notamment lorsque, dans un climat de crise politique et de désenchantement, entre l'épisode de la fuite du roi et l'été 1793, la légitimité des pouvoirs centraux a été assez généralement contestée. C'est alors que se développe (plus qu'il n'apparaît en 1791) le thème récurrent des bons Français de province opposés aux exaltés de Paris et à sa populace, sa dictature et ses jacobins dont les clubs affiliés sont les émanations néfastes. Contre tout cela, de 1791 à 1793, le discours de la presse porte que la province est le refuge des "vrais Français ». Pour prendre en exemple l'un des plus importants et des meilleurs journaux locaux, on lit dans la Chronique nationale et étrangère de Rouen des appels répétés à la sagesse de la province dont je ne citerai que trois exemples parmi bien d'autres :

«Le roi est rentré dans la capitale; tout est changé. Les écrivains incendiaires ont repris la plume [...], ils insultent le monarque et son épouse par des pamphlets virulents, et le peuple exalté se laisse entrainer à des vœux homicides [...]. France, éveille-toi, c'est à toi, à tes 83 départements réunis qu'appartiennent le droit de juger le prince » (30 juin 1791).

"Citoyens des départements, craignez Paris et ses conseils corrupteurs » (9 novembre 1791).

«Où est-il donc l'espoir présent de la patrie ? Il est dans les départements [...]. La monarchie vit encore dans les départements, les mœurs sont bonnes encore; la religion, surtout la religion, y est encore respectée; et c'est là qu'il y a vraiment des Français et des citoyens » (5 décembre 1791).

Le prospectus d'un journal lancé à Évreux en l'an III annonce :

"Intimement convaincus que Paris, cette immense commune, qui fut depuis le 2 septembre jusqu'au 9 thermidor, le foyer de tous les crimes, est encore malheureusement le centre de trop de passions, nous sommes venus, dans la simplicité de nos cœurs, proposer aux Départements la rédaction d'un journal philanthropique, soutien des principes éternels de la vertu et de l'humanité. [...] Puissiez-vous, citoyens des départements, agréer un Journal consacré spécialement à votre défense, à votre gloire même, puisqu'on se propose d'y démontrer qu'en tous temps, voulant le règne des lois, vous avez été les plus vrais et les plus purs républicains.»

(1) "L'image de la Révolution dans la presse provinciale ", dans L'image de la Révolution française, communications présentées lors du Congrès mondial pour le Bicentenaire de la Révolution, Sorbonne juillet 1989 (Michel Vovelle, dir.), Pergamon Press, 1989, pp. 140-145. 
Cet exemple est cependant tardif et la thématique récurrente des " vrais Français " de province tend nettement à régresser après son zénith de juin et juillet 1793, moment exceptionnel d'unanimité provinciale anti-parisienne, plus apparente que réelle d'ailleurs. Ce discours est un leurre qui a pu tromper les contemporains ou les historiens dans les interprétations à donner du fédéralisme, puisqu'il tend à faire croire, d'une part, qu'il y avait une opposition presque irréductible entre Paris et la province, et d'autre part, que cette dernière est uniforme dans sa résistance potentielle à la capitale. Mais il est lourd de conséquences quant aux processus de prise de décision politique car il me semble être à l'origine des politiques quasi obsessionnelles de formation et de direction de l'esprit public qui se mettent en place à la même époque, à partir de 1792, et précisément pour diffuser la bonne parole ou détruire la Contre-Révolution dans les départements. La presse locale donnerait en permanence, justement par l'importance faite à l'événement parisien, une image de la capitale en Révolution qui suscite des réactions de rejet ou de sympathiè, souvent interprétées par les gouvernements successifs comme une réponse à l'exigence d'unité nationale ou de constitution de cet « esprit public » auquel la province refuserait ou accepterait d'adhérer.

\section{Réseaux de distribution et aires d'influence de la presse locale}

Est-ce à dire que l'on a pu craindre le sursaut d'un patriotisme provincial ravivé à la veille de la Révolution par la presse d'Ancien Régime (2) ? Probablement pas : les clivages entre haute et basse Normandie, Bretagne intérieure et maritime, par exemple, signifient que dominent d'autres logiques de solidarités identitaires que l'appartenance à une province historique. En revanche, beaucoup plus prégnant est le fractionnement du territoire national en réseaux tissés autour des villes (3), dont la distribution des journaux permettrait une bonne approche topographique si nous disposions de listes d'abonnés. En leur absence, il nous reste l'inventaire du courrier ou la liste des bureaux de distribution dont deux feuilles du Bas-Rhin donnent l'exemple en l'an IV (4) [carte 1].

(2) Cf. Claude LABROSSE, «La région dans la presse provinciale ", dans La presse provinciale au $x$ xir siecle (Jean Sgard, dir.), Grenoble, 1983.

(3) L'expression aires d'influences engloberait un monde rural où la vie politique a, comme l'ont montré les travaux de Roger Dupuy, Georges Fournier ou Jean-Pierre Jessenne, une grande vitalité et surtout une réelle autonomie; le mot néseau convient assurément mieux pour désigner à la fois la campagne immédiatement proche et, au-delà, les bourgs ou chefs-lieux de canton situés sur les axes principaux de communication.

(4) Source : Jean-Piene KINTZ, «Alsace », dans Pierre ALBERT et Gilbert FEYEL, La presse départementale en Révolution (1789-1799), Cahiers de l'Institut français de presse n³, La Garenne-Colombes, 1992. 


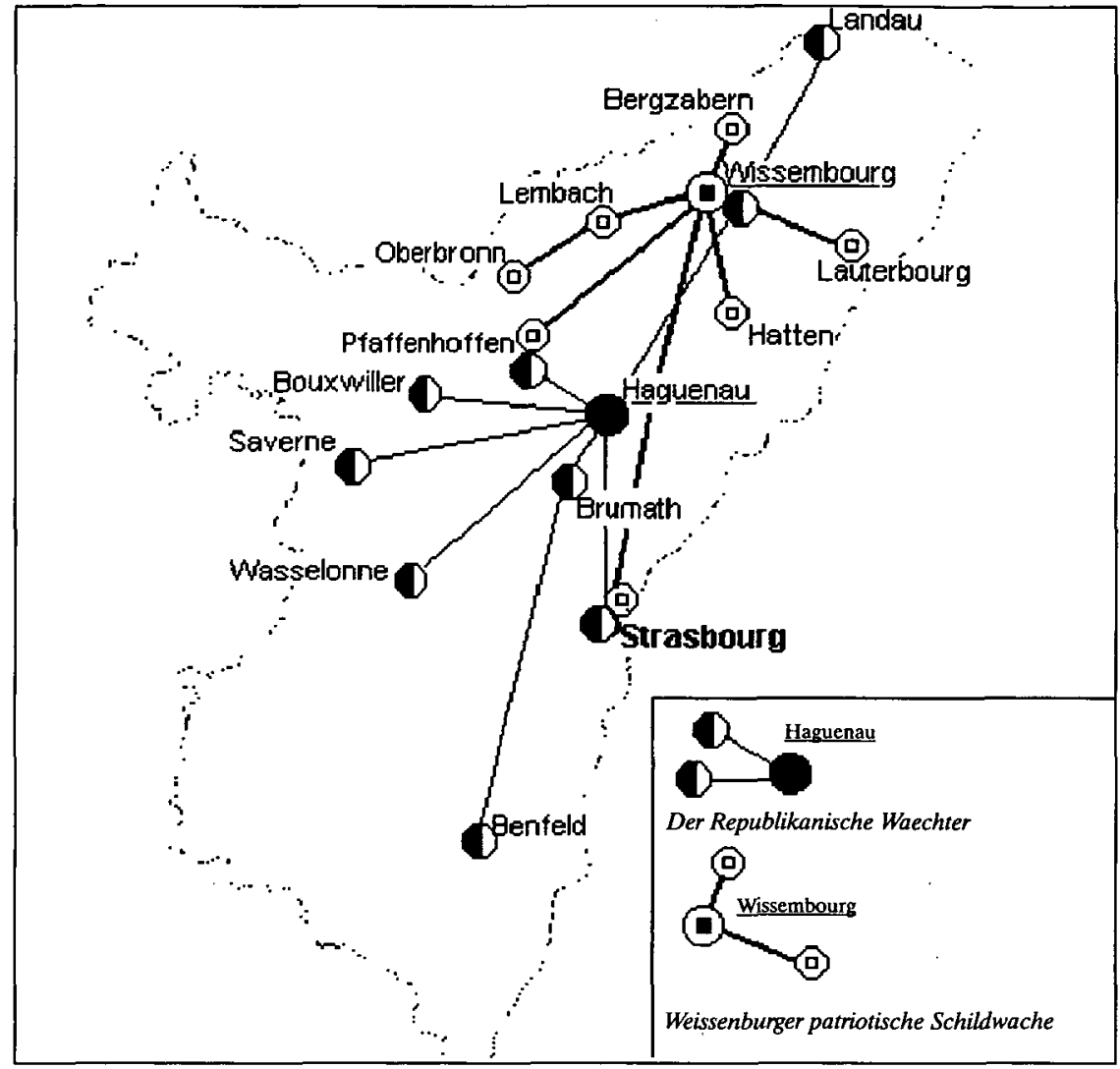

Carte 1 : bureaux de souscription de deux journaux du Bas-Rhin en l'an IV

Au-delà des limites départementales, la liste des bureaux de distribution est à examiner avec prudence : le Journal Patriotique de Pétradura lancé à Lisieux en 1790 annonçait être vendu «chez tous les libraires de Paris, Rouen et Caen, ainsi que chez ceux de Londres »! Plus vraisemblable est la distribution du Méridien de Rouen (1799) avec ses bureaux au Havre, à Dieppe, Yvetot, Montivilliers, Neufchâtel, Pont-l'Évêque, et plus loin à Cherbourg très vraisemblablement par Le Havre et la mer. Lorsque Babeuf lance Le Correspondant picard à Noyon en octobre 1790, son prospectus donne une liste impressionnante de villes où son journal est mis en vente; or, d'après V. Daline (5), une liste d'abonnés révèle que non seulement il y a des lecteurs dans chacune de ces villes, mais également ailleurs, et il était donc possible de distribuer (durablement?) une petite feuille sur trois départements [carte 2].

(5) Gracchus Babeuf à la veille et pendant la Grande Révolution française (1785-1794), 1976, cité par J. BERNET dans La Presse départementale en Révolution, op. cit., pp. 81-83. 


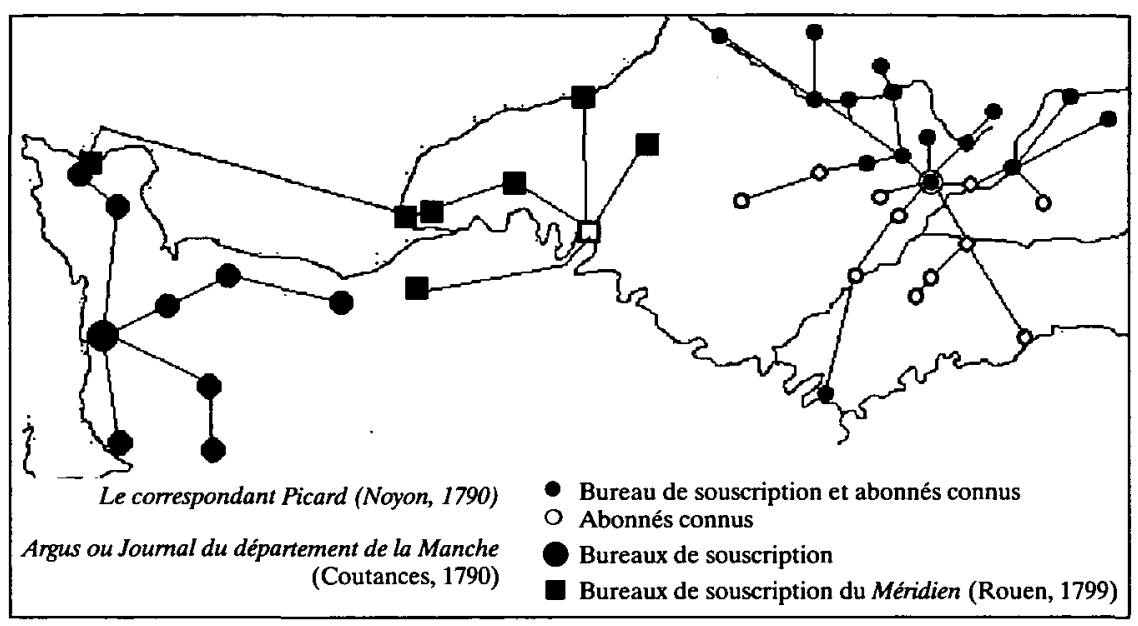

Carte 2 : trois réseaux interdépartementaux de distribution

En ne considérant que les bureaux extra-départementaux des journaux les plus solides (d'après leur durée de parution), la presse de Strasbourg et celle de Rouen couvrent des aires plus larges encore, mais. c'est là sans doute une extension maximale : les journaux de Rouen par exemple, à l'exception des grands organes royalistes, se distribuent globalement davantage à l'ouest qu'à l'est de la ville, ce qu'on peut expliquer par la concurrence de la presse parisienne et, plus généralement, par l'influence de la capitale. En effet, cette partition se retrouve peu ou prou dans la distribution des propriétés foncières dès la fin du XVIIe siècle, d'après des sources notariales, ou à la fin du XVIII' siècle, d'après les achats de biens nationaux. La presse n'est ici qu'une des manifestations d'un découpage du territoire national en fonction de logiques sociales et culturelles dont Michel Vovelle a montré la complexité dans sa Géopolitique de la Révolution française (6) et que confirme l'échec d'un mouvement fédéraliste lui-même atomisé en « fédéralismes » (7) [carte 3].

Les diverses instances des pouvoirs centraux, mais pas seulement elles, tous les acteurs engagés de la vie politique, se trouvent donc face à un dilemme : soit prendre en compte le morcellement de l'espace politique national en réseaux périurbains de solidarités, soit concevoir l'opinion publique comme la matrice d'une citoyenneté moderne, nationale et recom-

(6) Michel Vovelle, La découverte du politique. Géopolitique de la Révolution française, Paris, La Découverte, 1992.

(7) Cf. le colloque de Marseille consacré en 1993 au sujet : Bernard CousıN (dir.), Les fédéralismes, Aix-en-Provence, P.U. Provence, 1995. 


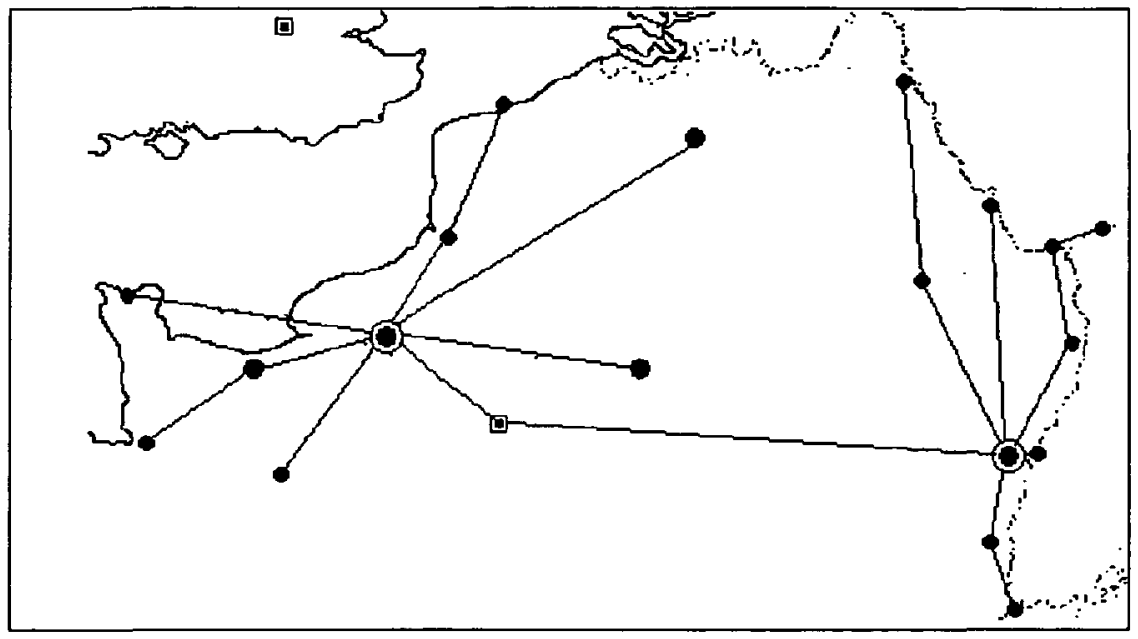

Carte 3 : bureaux de souscription des principaux journaux de Rouen et de Strasbourg, hors de leurs départements respectifs

posée en fonction de clivages nouveaux, idéologiques et universels, et donc ignorer, voire combattre ces intérêts particuliers fondés sur l'identité locale et nécessairement archaïques. En matière de presse, il s'agit de subventionner ou de créer un journal local, ou au contraire de diffuser des feuilles nationales et de constituer un réseau fédérateur de l'opinion. La seconde option me paraît avoir dominé, d'abord parce qu'elle était plus conforme aux idéaux de 1789 : on n'est pas très loin ici des débats de la Constituante (pensons au discours de Sieyès le 17 septembre 1789) sur la fonction même du député, porte-parole de la nation entière et non de ses seuls commettants. On peut d'ailleurs se demander si la départementalisation du territoire en 1790 visait à briser ces solidarités ou au contraire à les institutionnaliser. Toujours est-il que lorsqu'ils sont intervenus directement dans la presse locale, les pouvoirs publics nationaux ont inscrit leur action dans un cadre départemental qui s'est avéré inefficace, comme le révèle l'exemple de La Vedette Normande.

Ce journal reçut en l'an IV un soutien du comité de sûreté générale, «persuadé qu'il importait au bien public d'empêcher la chute du seul journal dans les principes républicains qui fût rédigé à Rouen » et le gouvernement prit un abonnement de 240 exemplaires " afin de vivifier l'esprit public et de rallumer dans tous les cœurs l'amour sacré de la liberté ». La Vedette devait être distribuée dans les chefs-lieux de canton du département, « dans les hôpitaux militaires, les cafés les plus fréquentés des lieux, surtout là où il y a marché » (8).

(8) Archives départementales de Seine-Maritime, L 304. 
L'expérience fut vite abandonnée, pour des raisons qui ne sont pas très claires. L'explication avancée officiellement est la diffusion difficile du journal. Mais faut-il mettre en cause les conditions matérielles de distribution, notamment dans le pays de Caux où simultanément arrivait très bien le réactionnaire Observateur de l'Europe, ou plutôt l'inadéquation entre les exigences territoriales du gouvernement (couvrir le département) et l'aire d'influence du journal? En effet, mal distribuée dans l'ouest du département, La Vedette atteint exceptionnellement le pays de Bray où son arrivée est une innovation notée à Argueil et Aumale, et regrettée après la fin de la subvention; mais elle était assez bien vendue (d'après le courrier) à Dieppe, le long de la Seine jusqu'au Havre, dans l'ouest de l'Eure et même à Caen. Il semble avoir été extrêmement difficile de déborder du réseau de distribution, du tissu de relations ou de connivence politique d'un journal au républicanisme beaucoup plus prononcé que celui du gouvernement.

Le cas de La Vedette n'est pas isolé : L'Abeille politique et littéraire de Charles de Limoges était, en 1790-1791, bien répandue entre Rouen, Le Havre et Pont-l'Évêque, tout autour du pays d'Auge; admettons que sa propagande réactionnaire et aristocratique ait trouvé là un terrain favorable. Or, après quelques vicissitudes, le ci-devant chevalier de Limoges, à peine sorti de prison, lance en l'an III, un Journal du Soir dont l'aire de distribution, est presque exactement la même, entre Caen, Le Havre, Lisieux et Louviers. Le Journal du Calvados, successivement monarchien, feuillant, girondin, puis royaliste, atteint Falaise, Bayeux, Pont-l'Évêque, Lisieux, donc presque tout le département à l'exception du district de Vire, bastion républicain, anti-fédéraliste et anti-chouan [carte 4]. L'aire de prédilection durable de chaque feuille obéit donc à des logiques politiques qui ne recoupent pas exactement le réseau d'influences culturelles ou économiques

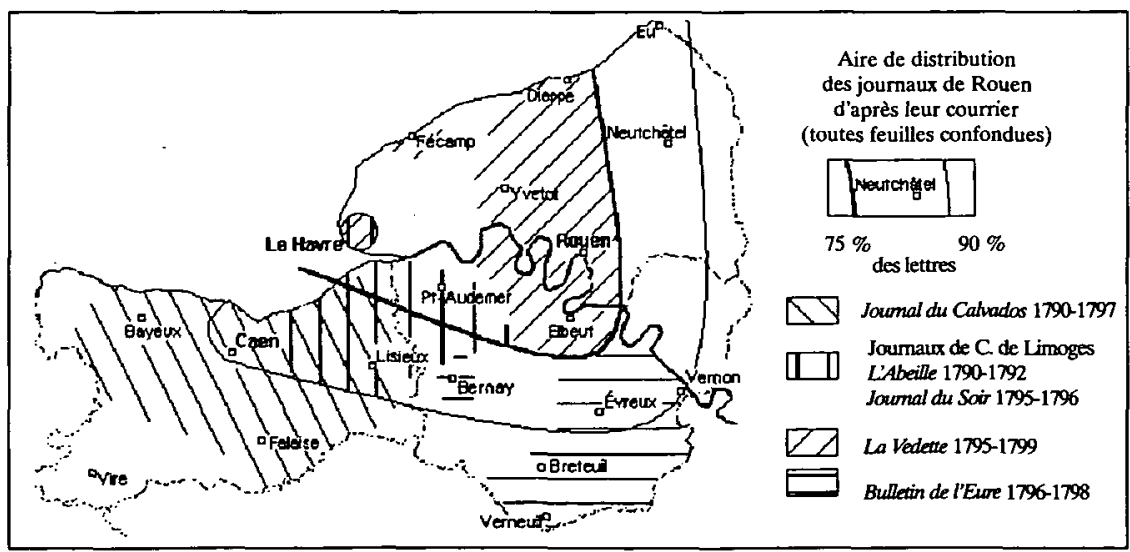

Carte 4 : aires de distribution de quatre journaux normands 
d'une ville et dépassent la seule défense d'intérêts locaux. On peut donc se demander si les groupes d'individus constitués autour de journaux locaux porteurs de valeurs idéologiques partagées, tendent à se fédérer en réseaux d'opinion à l'échelle nationale.

De ces réseaux nationaux, les journaux royalistes offrent un exemple peut-être marginal, mais spectaculaire, constitué autour de Magloire Robert. La longue carrière de ce journaliste commence à Fécamp où il crée un quotidien, à l'ombre du député Cherfils, qu'il rejoint ensuite à Paris. Il rédige alors, de mars à octobre 1793, L'Observateur de l'Europe dont les rapports de police soulignent la distribution à profusion, l'influence et le danger :

«Le rédacteur de cette feuille est évidemment un homme de parti. La commune de Paris, la Montagne, les Jacobins y sont peints sous les couleurs les plus affreuses. [...] Les événements, les opinions, tout y est tronqué, dénaturé : cette feuille paraît extrêmement dangereuse [...] L'espèce de fureur avec laquelle on se l'arrache, prouve à quel point, cette feuille échauffe et exalte certains esprits. » (9)

Des deux rédacteurs, dit-on, «l'un d'eux est député à la Convention nationale et l'autre de la Société des Amis de l'Égalité, aux Jacobins » (10). À la fin de 1793, L'Observateur étant interdit, Robert gagne Rouen où il rédige dans l'ombre la Gazette révolutionnaire, avec sa femme pour prêtenom. C'est sans doute à Rouen un secret de polichinelle : en thermidor, c'est par ce journal que le bureau permanent de la commune apprend la chute de Robespierre et, comme le courrier officiel tarde à confirmer l'information, on juge plus prudent de faire confiance à la Gazette révolutionnaire que l'on dénonçait un mois plus tôt. Tout cela laisse évidemment supposer que des liens solides existent entre Paris et le journaliste normand : correspondant à Rouen du Messager du Soir, Robert lui fait écho ou lui fournit de la matière. Pendant la réaction thermidorienne dont il est l'un des principaux acteurs à Rouen, il est président des commissaires de section chargés de pourchasser les terroristes; il est aussi aux premières loges en vendémiaire IV, lorsqu'il tente de soulever la ville contre la Convention. Mais le réseau Robert est plus étendu encore : ses feuilles couvrent toute la Normandie et débordent sur Paris, Reims, Amiens. À peu de choses près, la Chronique nationale et étrangère de 1790-1793 avait la même distribution, vers Paris ou la Somme. Dans le reste de la province, à l'exception du département de l'Orne, L'Observateur de Robert est recopié par le Courrier maritime du Havre et par le Journal général de Caen. Dans ce dernier titre, on lit le 5 thermidor an V

(9) Archives nationales, F1c III-Seine 27, 16 juin.

(10) Ibid., F7 3688/3, 16 septembre. 
que « tout semblait présager les plus violents orages [dans la capitale], mais trois lettres écrites de Paris à l'estimable rédacteur de L'Observateur de l'Europe nous rassurent pleinement ». Or, les relations entre le journal de Caen et le journaliste de Rouen étaient bien antérieures à cette époque puisque le Journal du Calvados du 21 juillet 1793 démentait le bruit selon lequel L'Observateur de l'Europe (alors parisien) ne paraissait plus depuis le 2 juillet.

Ce qui paraît donc évident, c'est l'existence d'un réseau royaliste avec un relais rouennais entre Paris et la Normandie, rôle tenu par la Chronique jusqu'au début de 1793, puis par les feuilles de Magloire Robert. Le discours provincialiste de la Chronique ne l'empêche nullement de s'appuyer sur un réseau très étoffé entre la Normandie et Paris où l'équipe rédactionnelle est solide. Enfin, l'abondance des nouvelles venues de Bordeaux dans L'Observateur n'est peut-être pas liée seulement à l'intérêt des négociants pour les activités portuaires : elle renvoie à une comparaison à faire entre les articles de la feuille rouennaise et le Nouveau Journal des Journaux, sachant que les deux rédacteurs, Robert à Rouen, Lawalle à Bordeaux, ont des carrières très semblables, ils rassemblent autour d'eux la jeunesse de leur ville, tel ce Homberg, fils d'un négociant havrais et un temps prête-nom de Lawalle.

\section{Relations interurbaines et circulation de l'information}

Il importe donc de comparer les articles entre deux feuilles de province. En fait, il faudrait faire beaucoup plus et je souhaiterais que cette communication soit perçue comme un appel à l'ouverture d'un chantier de recherches évidemment collectif. Les études, mêmes les plus récentes, du contenu rédactionnel de la presse de province ne prennent en compte que les informations locales, et pour le reste, se contentent de signaler la présence de "nouvelles de France et de l'étranger ", assorties parfois du commentaire peut-être prématuré : elles viennent de Paris. Or, si les journaux se recopiaient entre eux, et plus exactement si l'on pense que ceux de province plagiaient ceux de la capitale, je crois qu'il faut réviser notre vision d'un schéma pyramidal (de la capitale vers la province) de la circulation de l'information pendant la Révolution.

On sait déjà que les journalistes de province échangeaient leurs feuilles; ainsi pour prendre l'exemple du Journal de Marseille (11), Ferréol Beaugeard recevait 13 feuilles provinciales en 1789, 25 en 1791, 39 en 1793, et environ

(11) René GÉRARD, Un journal de province sous la Révolution. Le Journal de Marseille de Ferréol Beaugeard (1781-1797), Paris, Société des études robespierristes, 1964. 
deux fois moins de titres parisiens. Un test portant sur les dates des nouvelles de province données dans le Joumal de Rouen révèle de nombreuses anomalies qui laissent supposer que les informations arrivaient par la mer et qu'il existait un réseau atlantique, via Le Havre [carte 5]. Deux cas intéressants sont ceux de Toulouse et de Perpignan dont les informations étaient sans doute drainées par Bordeaux vers Rouen; ainsi, lorsque le Joumal de Rouen du 14 nivôse an II signale que «des circonstances particulières nous ont privés depuis près d'un mois de nos correspondances de Perpignan », est-ce parce que la pénurie de papier a suspendu la parution de L'Écho des Pyrénées du 5 au 28 frimaire, ou parce que la filière bordelaise était momentanément empêchée de fonctionner? Sur la carte de la France vue de Rouen, un grand arc occidental ou atlantique regroupe donc les villes dont les informations ne passaient sans doute pas par Paris, et il me semble que cela n'est pas sans conséquence sur la formation de l'opinion publique [carte 6].

Ces réseaux sont-ils de simples dispositifs rédactionnels en quête de nouvelles dont la crédibilité serait garantie par l'ancienneté d'un journal ou la solidité d'une équipe rédactionnelle étoffée, ou bien répondent-ils à des affinités politiques électives? Le Journal de Rouen, par exemple, donne peu de nouvelles d'Évreux, sauf en 1797-1798, c'est-à-dire au moment où paraît le Bulletin de l'Eure de Touquet, à la confluence de deux opportunités de nature différente : la parution d'une feuille dans l'Eure permet le drainage des nouvelles et il y a convergence politique de deux journaux unis ainsi dans la lutte contre les royalistes et dans la propagande en faveur de la création de cercles constitutionnels.

La presse offre sans doute un moyen de déceler les liens unissant la province à la capitale, d'une part, les départements entre eux, d'autre part. Cela pose des problèmes de méthodologie et exige surtout la mobilisation de nombreux chercheurs. Il est vrai que les sources sont rarement mentionnées par les journalistes, et quand elles le sont, ce sont souvent les plus politiquement correctes du moment. Mais pas toujours. Les Annales de la Révolution en 1794 citent régulièrement le Journal des 3 décades, L'Antifédéraliste, Le Père Duchesne, les Affiches d'Angers, le Journal républicain de Marseille, Le Batave, L'Abréviateur de Tours, ensemble assez disparate dont il est difficile de tirer des enseignements, sauf si l'on relève que le Joumal des Hommes Libres est, de loin, le plus largement reproduit. La Renommée ou Journal de Mortagne (fructidor an IV-brumaire an VI) a des sources plus homogènes : les Annales de la République française, la Gazette nationale de France, La Quotidienne, le Journal de Perlet, L'Ami de la Patrie (12). Dans les deux cas, il

(12) Mais pas L'Observateur de l'Europe pourtant bien distribué dans les quatre autres départements normands. 


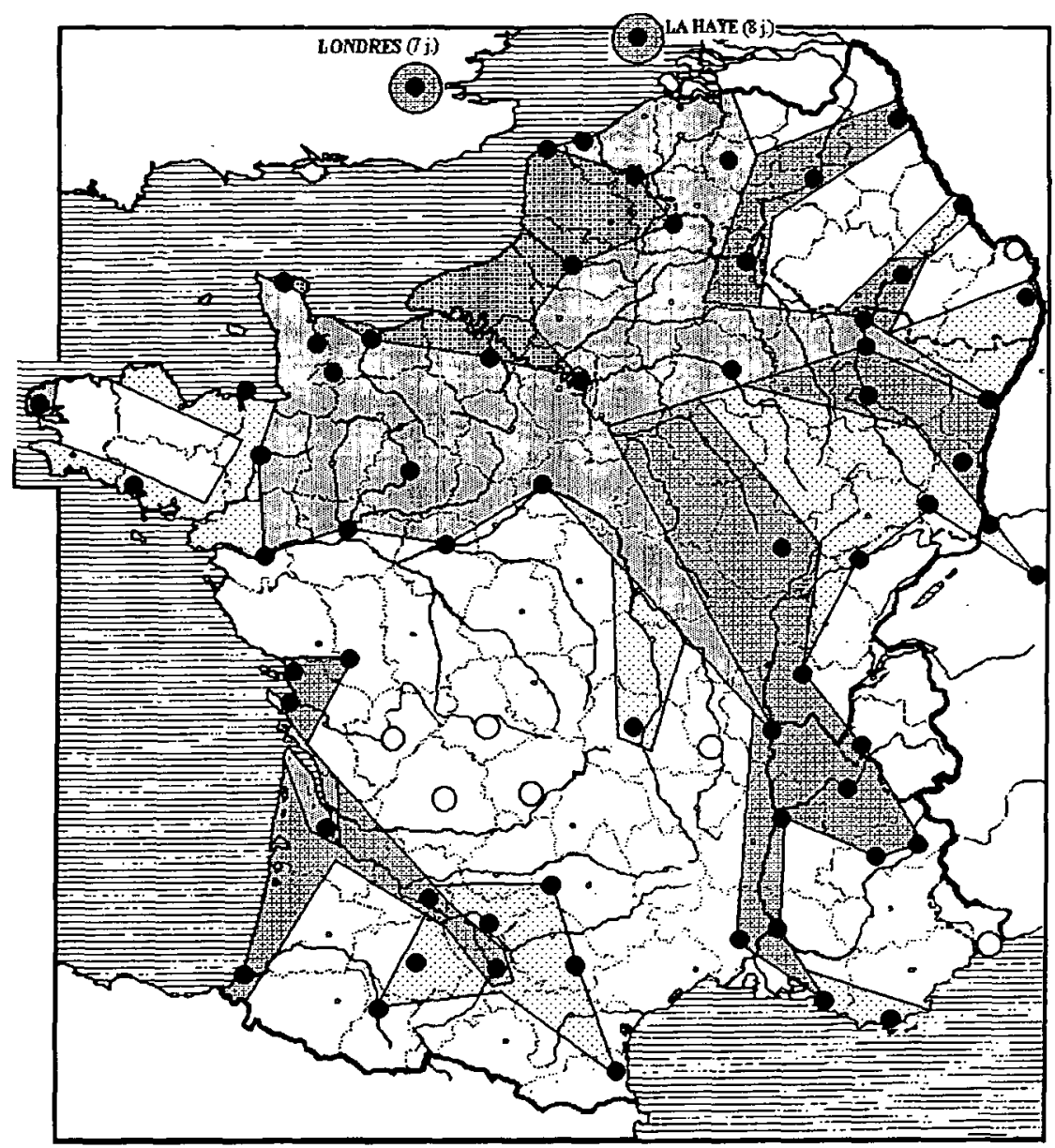

Carte 5 : délais entre la date des nouvelles de province et celle de leur publication dans le Journal de Rouen, du 1er décembre 1790 (il devient quotidien) au 18 brumaire an VIII (minima observés au moins trois fois)

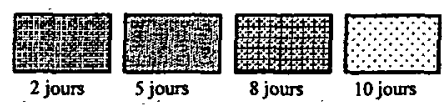

Remarques : au temps moyen (avec de grandes variations) on a préféré les minima observés au moins trois fois (ainsi pour Lille ou Valenciennes : 2 jours, Bruxelles ou Strasbourg : 4, Bordeaux, Nantes, Lyon : 5 , Toulouse : 7, Caen : une journée). Perpignan est à 10 jours, mais des délais de 9 et même 8 jours ont été observés (deux et une fois).

Ces délais sont variables au cours de la période : de l'an III à l'an VI, trois jours de Dunkerque à Rouen, mais avant comme après ces dates 8 jours (probablement par Lille et non plus par la mer).

Les nouvelles d'Orléans peuvent arriver (en' 4 jours) par Évreux ou Paris; la seconde option paraît plus vraisemblable.

Les données sont insuffisantes pour le centre Ouest (où il y a d'ailleurs peu de journaux locaux), la Bretagne intérieure (délais le plus souvent supérieurs à 10 jours) et le centre Est ; on a indiqué quelques villes d'où les nouvelles sont venues plusieurs fois, en plus de 10 jours: 


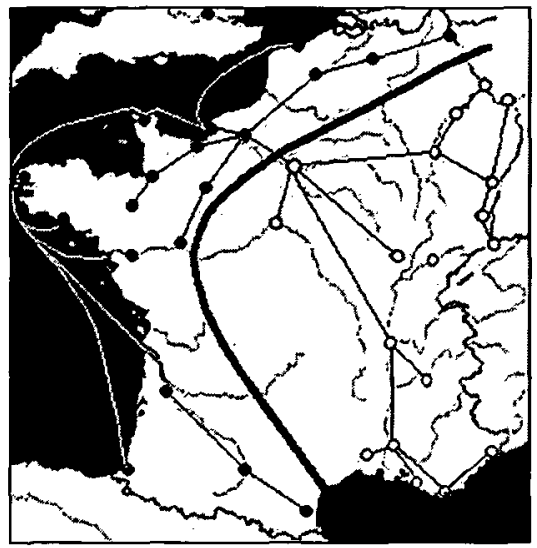

Carte 6

France Atlantique et France intérieure (Nouvelles passant par Paris)

serait utile de repérer les titres les plus fréquemment, voire systématiquement utilisés. Les annonces publicitaires de journaux parisiens dont le bureau de la feuille locale assure la souscription sont peut-être une autre piste.

Comparer les nouvelles locales, les articles d'un titre à l'autre, le chantier est immense, mais il révélerait sans doute que la place de Paris dans la presse provinciale n'exprime pas seulement l'importance des événements qui s'y déroulent ou le poids politique de la capitale. La presse est surtout l'agent et le reflet, plus ou moins fidèle, de la formation d'une opinion publique à l'échelle de la Nation entière : elle diffuse une information essentiellement nationale, donne aux conversations des sujets communs et tend ainsi à l'unification de l'espace public théorisé par J. Habermas. Elle tend aussi à créer de nouveaux groupes qui se superposent plus qu'ils ne se substituent aux solidarités locales traditionnelles dont il convient d'ailleurs de ne pas exagérer la prégnance : l'événement révolutionnaire engendre la création de courants politiques véritablement nationaux autour des mêmes sensibilités idéologiques ou des mêmes intérêts de classes.

L'échec de La Vedette normande traduit sans doute à la fois l'importance des nouveaux clivages de l'opinion et le refus d'une presse subventionnée aux ordres du pouvoir, en somme cette exigence affirmée depuis 1789 d'indépendance de la presse qui doit rendre compte des enjeux nationaux même si elle le fait par une relecture locale. Les tentatives de subvention se soldèrent très majoritairement par des échecs. Mais cela ne signifie pas refus du national : la presse participe à l'élargissement de la discussion citoyenne, et les journaux purement locaux sont rares, éphémères et souvent datés du début de la Révolution, telles ces feuilles destinées en 1790 ou 1791 à préparer les élections locales, qu'on ne trouve plus ensuite (13). Sous le Directoire, la conscience des enjeux nationaux est plus évidente, ce qui nous

(13) Il est d'ailleurs possible que le lancement de ces journaux à vocation territoriale très limitée ait visé à tisser un lien entre les citoyens d'un même département et la petite ville promue capitale politique locale mais jusqu'ici dépourvue de rayonnement. C'est peut-être là l'explication de la démesure constatée dans la distribution annoncée des petites feuilles évoquées plus haut, celle de Lisieux ou l'Argus de Coutances (cf. carte 2; Hugues PlaideuX, "L'Argus, premier journal du département de la Manche [Coutances, juin-juillet 1790]", Revue de la Manche, t. 42, 2000). 
ramène à la question de la formation de réseaux politiques, de dimensions variables, plus ou moins ancrés localement, mais fédérés ou en cours de fédération, avec cette interrogation que l'étude de la presse pourra peutêtre aider à résoudre : comment ces réseaux s'articulent-ils entre eux ou avec la capitale, pour constituer un courant d'opinion nationale?

Le «réseau atlantique » observé entre Rouen et Bordeaux pose deux questions : d'abord celle de son exemplarité; ensuite celle de sa relation possible avec quelques tentatives ministérielles de contrôler la presse : immédiatement après avoir soutenu la création du Joumal de Guyenne (en 1784), Vergennes appuyait celle du Joumal de Normandie, avec, dans les deux cas, une dépossession des propriétaires des anciennes Affiches et l'aide des intendants. Or, le Journal de Normandie était, sur les colonies ou le libéralisme économique, assez proche des positions politiques du ministère. On pourrait décliner son évolution ultérieure en trois temps :

1 - Devenue Joumal de Rouen, la feuille refuse, après Varennes, de cautionner la thèse de l'enlèvement du roi et peu après de relater la révolte de Saint-Domingue. À l'époque de ce virage politique, à la fin de 1791, est lancé à Rouen le Journal du Commerce dont le correspondant parisien Lemoine, membre de la Commune de Paris, s'affiche ennemi de «la secte meurtrière et visiblement soldée, pour assurer la perte de nos colonies ». Ce nouveau titre reste confidentiel et donc inutile...

2 - Mais, en janvier 1792, on apprend le changement de propriétaire et de rédacteur du Joumal de Rouen, l'arrivée de Noël de la Morinière qui huit jours avant cette promotion annonçait encore son prochain départ pour Göteborg, comme agent de la Marine de France ! Et dès le premier numéro qu'il rédige, il prend la défense des intérêts coloniaux, s'élevant contre les «philanthropes » qui font obstruction à l'envoi de troupes aux îles : « Nous diront-ils encore que nous pouvons nous passer de colonie et que le sucre n'est pas nécessaire? "

3 - Après le 10 Août, le Journal de Rouen devient ouvertement, sinon sincèrement jacobin. Curieusement, le Journal du Commerce qui végétait connaît un bon succès si l'on en juge par ses livraisons surprenantes : 16 à 24 pages in $-8^{\circ}$ chaque jour pour un prix modique.

Entre les deux feuilles, le ministère de la Marine paraît donc avoir balancé. Ainsi, les Annales de la Révolution puis La Vedette normande qui font suite au Journal du Commerce interdit au début de 1793, bénéficient encore de la caution d'imprimeur de la Marine, jusqu'en floréal an V. À cette date, le Joumal de Rouen reprend la main : on peut s'abonner chez Noël au Joumal historique et politique de la Marine et des Colonies, qui «déploie aux regards étonnés le système affreux qui a ruiné les îles françaises" et paraît avec cette épigraphe : «Point de Colonies, point de Commerce; Point de Commerce, plus de Marine ». Et jusqu'en ventôse an VI, il donne régulièrement des articles intitu- 
lés « Ministère de la Marine »... Jusqu'en ventôse an VI : c'est-à-dire jusqu'au moment où il entreprend avec les néo-jacobins d'Évreux de soutenir la création des cercles constitutionnels et de préparer de bonnes élections...

Il est difficile d'en dire davantage, sauf à remarquer que Guilbert, l'ancien rédacteur du Journal de Commerce, fortement menacé au début de 1793 a pu trouver refuge à Genève; que, revenu d'exil, il vit à Paris d'où il dirige sa feuille rouennaise, que ses ennemis royalistes prétendent que «c'est à Bailleul, Louvet et Chénier qu'il doit son retour en France " (L'Observateur du 11 floréal an V), qu'il serait revenu à Rouen les poches pleines de «l'argent qu'il a reçu pour écrire en faveur du gouvernement " (L'Observateur, 24 germinal an V). Pourtant, tandis qu'il est «imprimeur de la Marine», le ministre de la Police dénonce sa Vedette normande. S’il y a bien des réseaux ministériels dont il sera sans doute très difficile de trouver la trame, ils ne travaillent pas tous dans la même direction politique.

Au total, tout cela traduit la complexité d'une vie politique qui tend à s'organiser à l'échelle nationale, non pas en imposant une centralisation mais en fédérant les réseaux locaux préexistants selon des clivages nouveaux. N'est-ce pas au fond ce que tentent de faire les jacobins? Entre les deux tactiques politiques que j'ai évoquées plus haut, s'appuyer sur le local, option peu conforme à l'esprit de 1789, ou l'ignorer pour ne considérer qu'une logique nationale, une et indivisible, il me semble que les Jacobins, réputés centralisateurs, ont pourtant adopté la première, si l'on considère les feuilles lancées en 1791 et 1792 par les clubs affiliés. Par exemple, le Courrer républicain du Calvados, journal de la Société des Amis de la Constitution, entre en relation avec des feuilles de l'Ouest (14); il reproduit, sur deux colonnes, le prospectus de la Vedette ou Joumal du Département du Doubs par la Société des Amis de la Constitution. On peut d'ailleurs s'interroger sur les cheminements possibles de l'information entre deux journaux de clubs de province, « en droiture» ou avec relais parisien (le club ou le journal des Jacobins), par échange de journaux ou de correspondance :

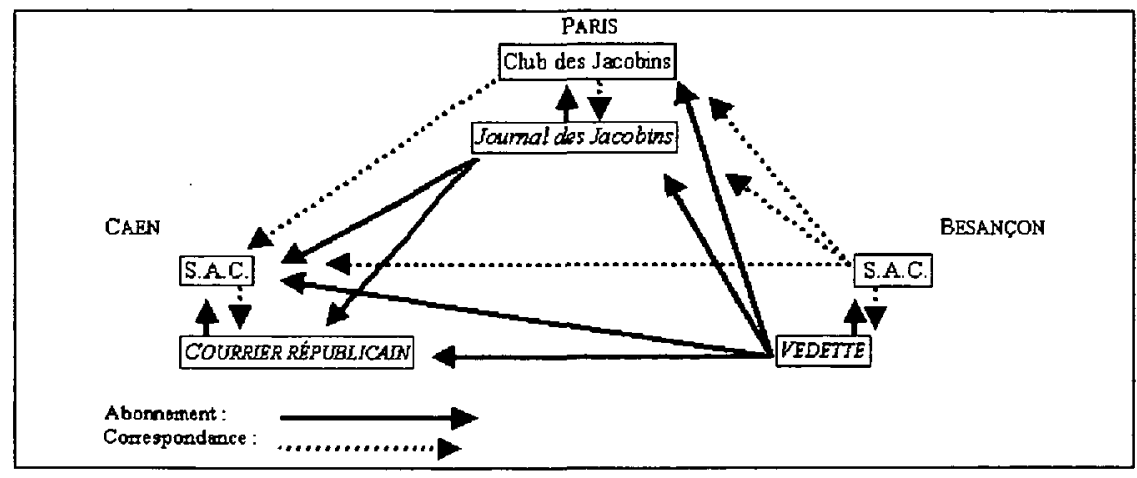

(14) On peut ainsi y lire, les 22 et 25 septembre 1791, une adresse de la Société patriotique de Brest. 
De même, en l'an II, la création de journaux par des représentants en mission, les subventions à des journaux locaux, la dynamique retrouvée des sociétés populaires cherchant à nouveau (après les tentatives de 1791-1792) à imiter l'exemple des Jacobins de Paris et à se donner un journal (15), tout cela paraît traduire une volonté de s'appuyer sur les solidarités locales, mais en fédérant les énergies. Il y a, à ce titre, un exemple sur lequel il faudrait sans doute plus d'informations : à Marseille, les représentants en mission apportent leur soutien au nouveau Journal républicain; parmi les rédacteurs, Ricord et Mittié sont ensuite à Perpignan où ils créent L'Avant-garde de l'Armée des Pyrénées Orientales. En messidor, Ricord est envoyé dans le département de l'Hérault, Mittié en Gironde où il lance la Chronique de Bordeaux, avant de quitter la ville précipitamment, rattrapé par son passé... Tout cela constitue peut-être une série de coïncidences et l'itinéraire des deux hommes serait à mieux connaitre. Il me semble pourtant que cela pose la question de la dynamique de formation d'une opinion publique nationale, celle du balancement des différentes instances du pouvoir entre deux tactiques politiques, en particulier de la politique des Jacobins, plus pragmatiques peut-être qu'il n'y paraît, dans une conception de l'unité nationale qui signifiait non pas uniformiser, mais fédérer, créer un journal ou un club pour former localement l'opinion publique et préparer son rassemblement ultérieur.

Il reste que le temps est venu d'un élargissement de perspective dans l'étude de la presse, trop cloisonnée jusqu'ici. Seul un chantier d'envergure nationale permettrait de dépasser le dualisme réducteur d'une dialectique provinces - Paris, et de cerner, au-delà de la vie politique locale, la constitution de réseaux nationaux d'opinion. Chantier immense, je le répète ; l'étude de la presse néo-jacobine, à la fois parisienne et provinciale de l'an VI, ou la comparaison des nouvelles et de leur circulation entre deux ou plusieurs villes (je pense à Bordeaux, Toulouse, Marseille, Lyon ou Strasbourg, puisque le relais de poste parisien n'est pas nécessaire), constitueraient par exemple des objectifs limités qui serviraient de premiers tests avant un travail de plus grande ampleur. Et Mac Luhan venant au secours de Gutenberg, il va de soi que la création d'une banque de données concernant l'ensemble de la presse nationale et provinciale serait indispensable.

Éric WAUTERS

Université du Havre

42, allée Mercure

76230 Bois-Guillaume

Eric.WAUTERS@wanadoo.fr

(15) É. WAUTERS, « La presse départementale en l'an II »,A.H.RF., 1996, pp. 1-36. 